or its alloys. It emphasizes the difficulties of preparing the metal in a state of high purity, and also the lack of knowledge about the really pure metal. It is well established that the temperature variations of some properties, such as the electrical resistance, undergo abnormal and quite unexplained changes about room temperature, while the apparently unrelated change from brittle to ductile fracture occurs between $50^{\circ}$ and $100^{\circ} \mathrm{C}$., and is of extreme interest. The physical properties of transition metals such as chromium and their alloys are, in fact, a direct challenge to both theoretical and experimental workers, and a reading of this book will suggest many subjects for research.

W. Hume-Rothery

\section{A THEORY OF SOCIAL CHANGE}

\section{Political Systems of Highland Burma}

A Study of Kachin Social Structure. By E. R. Leach. Pp. xii +324 . (London: The London School of Economics and Political Science (University of London), and G. Bell and Sons, Ltd., 1954.) 35s. net.

$\mathrm{T}$

HIS book, an account of the social structure of the Kachin-Shan population of north-east Burma, has a value quite independent of its descriptive content. To-day a very powerful and extensive school of anthropologists adopts what is known as the 'functionalist' approach. Institutions are thought of as functioning within a social structure which, itself, consists of individuals who are so connected up by social relations as to form an integrated whole. The social system is a functional unity and vice versa - the functional unit is a social system. All institutions support one another, all mutually determine one another, and the result will be the picture of a society in stable equilibrium.

Dr. E. R. Leach attacks this theory root and branch. He is interested in cultural change, and he frankly confesses that he finds no place for a theory of internal change in the functionalist approach. Why, he argues, should we suppose that equilibrium is 'normal'? Why should we even suppose that the societies hitherto pictured by functionalists as being in stable equilibrium really are so ? On the contrary, there is every reason to suppose that these societies do change, that they are at any given moment in a state of flux. In fact, Dr. Leach goes on, the functionalists do not and have not described the societies as they really are. What they have produced as a true picture of these societies is, in fact, nothing but a highly intellectualized conceptual model; and the reason for this, to his mind, is that they are attempting to impart a precision into the mutual relationships of individuals in such societies which in fact does not exist.

The anthropologist is using a set of scientific, highly precise and highly abstract categories, and into these he is trying to fit the data of native life; but in fact the native himself does not express his social relationships except in a symbolic and highly inconsistent form. He expresses it, that is to say, through ritual. He expresses it through myth. These specific and concrete activities are, moreover, interpretable in many ways, and individuals manipulate them to suit their own self-interest and in particular their interest in acquiring power and authority. In this way the system of myth and ritual is ambiguous enough to permit shifts of power-structure and to legitimize such shifts of power-structure once they have occurred. Thus Dr. Leach would (I think) hold that only unless an anthropologist is prepared to take as his assumption the vagueness and inconsistency of myth and ritual can he advance an explanation of indigenous political and social change. If myth and ritual were as tidy as the functionalists' assumptions tend to make them, then there is no room for shifts of power and status within the system described. It is only when myth and ritual are regarded as being a sort of arsenal from which legitimizing authority can be extracted that a theory of indigenous change becomes possible at all.

The major part of the book is devoted to an attempt to work out this theory in terms of the Kachin-Shan population. Here again Dr. Leach is an iconoclast: he admits blandly that his own interpretation of their cultures is at variance with practically everything that has hitherto been published.

His view can be briefly put in the following way : we have here three sub-types of social structurethere is the Shan type, which is feudal and hierarchical ; the Kachin gumlao type, which is equalitarian; and in between them, the Kachin gumsa type, which he maintains is in unstable equilibrium. This is a sort of cross-roads. It represents societies which are moving either from the gumlao to the Shan type or from the Shan type to the gumlao type. The gumsa type may move in either direction; its relations are not fixed. "I assume," he says, "that within a somewhat arbitrarily defined area-namely, the Kachin Hills Area-a social system exists. The valleys between the hills are included in this area so that Shan and Kachin are, at this level, part of a single social system. Within this major social system there are, at any given time, a number of significantly different sub-systems which are interdependent. Three such sub-systems might be typed as Shan, Kachin gumsa, Kachin gumlao. Considered simply as patterns of organisation, these sub-systems may be thought of as variations on a theme. The Kachin gumsa organisation modified in one direction would be indistinguishable from the Shan; modified in another direction it would be indistinguishable from Kachin gumlao. Viewed historically such modifications actually occur and it is legitimate to speak of Kachins becoming Shans or of Shans becoming Kachins. When therefore I, as an anthropologist, examine a particular Kachin or Shan locality I need to recognise that any such equilibrium as may appear to exist may in fact be of a very transient and unstable kind.'

This thesis Dr. Leach pursues through material of great intricacy. Yet is this single example, drawn as it is from one particular area, typical of all situations? Prima facie, one would not think so. It is perfectly possible, and indeed highly probable, that certain communities are in fact in the state of equilibrium. On the other hand, it must be conceded that it was high time that a field anthropologist put his finger on a crucial difficulty in functionalist theory-that is, the great problems it raises in constructing theories of indigenous change. The importance of this book therefore stands independently of the truth or falsity of Dr. Leach's proof. Its major importance is as a welldeserved challenge to a dominant anthropological theory.
S. E. Finer 